\title{
Shared Staff in the Hospitality Industry in the Postpandemic Era: Models, Strengths, and Challenges
}

\author{
Songting Zhang \\ School of History, Culture and Tourism, Fuyang Normal University, Anhui, China \\ Sol International School, Woosong University, Korea
}

\begin{abstract}
In the postpandemic era, with the change in the characteristics of hotel market consumption and the employment awareness of employees, the human resource management of hotels is facing challenges in terms of the employment model, labor relations and other aspects. The shared employment model is an effective practice for the hotel industry to innovate human resource management. By analyzing the concept of shared employees and profoundly understanding the relationship between the main elements based on research on the hospitality industry to share employee model, this paper discusses how in the postpandemic period, the hotel industry can share employees' strengths, challenges and coping strategies. The study shows the following: shared labor is an innovation strategy for human resource management in the hospitality industry and realizing an innovative model for flexible employment. The shared employee strategy constitutes a new form of win-win cooperation among hotels, society and employees. Hospitality industry-to-hospitality industry (H2H) and employee-to-hospitality industry $(\mathrm{C} 2 \mathrm{H})$ are the mainstream models of shared employment in the hotel industry in the postpandemic era. Implementing a shared employee management model in the hotel industry can help hotels reduce costs and increase efficiency, raise employment and employee incomes, and stabilize the social economy. In the future, the government, the industry, individual employees and other forces should be integrated to cooperate.
\end{abstract}

Keywords: Hospitality industry, Shared staff, Model, Advantages, Challenges.

\section{Introduction}

In early 2020, the COVID-19 outbreak traumatized the hospitality industry, bringing the industry to an instant standstill. To solve the problem of a large proportion of human resource costs, many hotels have laid off staff, and managers have reconsidered the existing employment model. The normalization of environmental changes caused by the pandemic, coupled with the fact that hotels themselves are more obviously in the off-peak season and an idle time, the hotel shared employee model of "borrowing soldiers" and of staff "crossing borders" provides a new idea for hotels to strategically innovate human resource management.

In the context of the sharing economy, forms of human resources classified as outsourcing, alliances among employees and part-time employment for some positions implemented in the service and hospitality industry are emerging forms of flexible employment (cf. e.g., Feng Xiliang, Zhang Jianguo, Zhan Jing, et al., 2018). In 2009, it was reported that the concept of the "gig economy", which aims to promote the value reconstruction and improvement of idle labor (Petriglieri G, Ashford S J, Wrzesniewski A. 2018: 124-170; Chen Xiangdong, Zhang Feng, Paul M.A. Baker. 2020: 21-29), had appeared. As early as the beginning of the 20th century, foreign scholars began to explore topics such as temporary workers, independent contractors, part-time workers, self-employment, gig workers, and freelancers. From the 1970 s to the 1980 s, concepts such as telecommuting, flexible employment and flexible employment gradually emerged. In the 1990s, research emerged on human resource outsourcing, external personnel, informal employees, labor dispatch, borderless work (borderless occupation) and other related topics. Since 2014, studies on the gig economy and other related topics have gradually emerged (Friedman G. 2014: 171-188). In recent years, the flexible employment model in the era of artificial intelligence has become the mainstream employment model in developed countries such as those in Europe as well as America, Japan and South Korea. In contrast, flexible labor practices and related theoretical research started late in China. After the outbreak of COVID-19 in 2020, there were studies on the path of "shared labor" for new parties of hotel self-help (Zhou Xuan, 2021: 26). Accordingly, this study systematically organizes shared employee models in the hotel industry, analyzes the advantages and challenges based on the macro environment, the hospitality industry and employees, and proposes countermeasures.

\section{Related Concepts}

\subsection{History of Shared Employees}

As early as the Qin Dynasty in China, there were peasants doing part-time writing and painting for others. In modernity, there have also been temporary workers in the vast rural areas during the busy farming season. In his book "Shouting · Hometown", Lu Xun wrote, "There are three kinds of people who work for us here: those who work for a certain family all the year round are called permanent workers. Those who work by day are called day laborers. They also farm and work for certain families only during the Spring Festival, holidays and rent collection". It was seen that the early years of part-time employment mostly involved labor transfer to pay the actual labor to get revenge.

In the context of the internet, especially after the emergence of various platforms, part-time jobs break through the restrictions imposed by time and space, and the types of jobs are becoming increasingly diversified, expanding from the transfer of physical strength to the transfer of professional knowledge, professional skills and experience output. 


\subsection{Shared Employee Concept}

There is no consensus on the concept of a shared workforce. Based on the diversity of current views, shared employees can be defined as follows: there can be different employment subjects to adjust for the particular time or period of the periodic employment imbalance. Shared employees are based on the willingness to respect employees, multilateral consensus and the not-for-profit premise. Cross-border sharing will occur for idle human resources to realize the socially optimal allocation of human resources among staff suppliers and demanders, and waiting staff can achieve a new cooperative win-win labor model (He Jiang, Yan Shumin, Guan Jiao, 2020: 1-13).

In a narrow sense, the shared employee model is a flexible way to adjust the surplus and shortage of human resources among enterprises. In a broad sense, it is a kind of adjustment and sharing of labor resources among different labor subjects such as enterprises and individuals.

\subsection{Principal Elements of Shared Employees}

The shared employee model includes three main elements: the employee supply side, employee demand side and individual shared employee.

First, enterprises with shared employees should make differentiated choices to avoid facing periods of weakness and prosperity during cooperation. In addition, in a special period, different qualities and the crossover of the labor subjects will lead to the matching of the labor shortage side and the corresponding surplus side to realize the allocation of the human resource surplus and shortage among different labor subjects.

Second, individual shared employees can come from inside or outside the enterprise (associated businesses, third-party human resource companies, etc.). Importantly, individual shared employees have multiple work skills or simple skills that can be employed by short-term training, and they have spare time to provide services for a variety of occupations. Some related positions involving high skills or trade secrets are not suitable for sharing even if the employees who hold such positions are idle.

According to the "Shared Staff Data Report" released by the Part-Time Cat, shared staff mainly come from five industries, i.e., traditional catering, hotel tourism, e-commerce, advertising and education, among which the flow of shared staff in and out of traditional catering and hotel tourism is high.

\section{The Rise of Hotel Shared Employees}

\subsection{Reasons for the Rise of Hotel Shared Employees}

\subsubsection{Institutional guarantee}

In China's Labor Contract Law, the factor of "sharing" has gradually been highlighted, and the concept of "sharing" has gradually been liberalized, which further promotes the breakthrough of the model of shared employees from explicit "prohibition" to implicit "permission". During the COVID-19 period, the state and local governments provided various special policy supports to facilitate the resumption of work and production, which further stimulated and developed the shared employee model.

\subsubsection{Macro environment}

(1) The change in the economic structure and the demand of the off-peak season of the hotel industry have laid the market environment foundation for the shared employee model. China's tourism economy has entered a stage of high-quality development, and the labor force in the service and hospitality industry has also shown structural changes. Hotel staff, especially bottom-level staff, have a high turnover rate, bottom-level positions have an obvious off-season and peak season, and the contradiction between supply and demand has surged. New economic forms such as artificial intelligence and digitalization have led to the transfer of jobs and the unemployment of people in middle- and low-end positions. Sharing flexible employment methods makes it possible to cope with the shortage of talent and to stabilize employment.

(2) The popularity of artificial intelligence technologies such as the internet and big data provides a convenient basic guarantee for hotels to share staff. Artificial intelligence employment platforms, big data analysis and accurate matching enable both supply and demand to obtain information efficiently, improve the efficiency of personnel screening, and reduce the cost of sharing transactions. Without affecting the original system of the division of labor, a third-party platform can refine the employment form, accurately match supply and demand information, and fully stimulate the individual potential of workers on the basis of social and economic stability.

(3) In the context of the sharing economy, the trends of flexible employment and service outsourcing have provided the audience base for shared employees in the hotel industry. For the tourism industry, which is prominently in a light boom period, new business concepts such as flexible employment and service outsourcing have long been accepted by employees. The model of flexible employment and flexible employment has become the new normal of the tourism industry. The implementation of employee sharing in the hotel industry is a strategic innovation of human resource management under the pandemic situation.

\subsubsection{Hotel demands}

At present, the diversified development trend of China's service and reception industry is obvious, and the boundary is increasingly blurred (Guo Z G., 2007: 60-64). The concept of employment has changed from fixed to flexible, and forms of shared employment can be easily promoted and implemented in the hotel industry. On the one hand, in recent years, rising labor costs, the loss of bottom-level staff and other prominent labor difficulties in the hotel industry have encouraged hotels to share labor and staff for cross-border work in organizational management. The nature of the work in hotels determines that it is obvious when hotels are busy. Sharing the input of staff becomes an effective means of personnel replenishment. On the other hand, in the context of the 
pandemic and during the off-season, idle hotel employees can be exported and shared, which also makes it possible for hotels to reduce their human resource costs. In addition, based on the long-term strategy and considerations of social responsibility, hotels have begun to share employment and implement other flexible employment methods at the level of human resource strategy.

\subsubsection{Employee demands}

From the perspective of individual employees, the change in employment ideas, the driving factor of economic interests and other factors encourage them to gradually accept this flexible and free employment form. Individual employees also hope to relieve the economic pressure of "being too busy" in special periods such as COVID-19 as well as the work pressure of "being too busy to death" in special periods of the hotel through the form of shared employment. Shared employment also provides an opportunity for individual employees to enrich their career and cross-border training.

\subsection{The Basic Model of Shared Employees in the Hotel Industry}

Based on different sharing subjects, the Hefei Municipal Human Resources and Social Security Bureau proposed three shared employee models: a coordination model of human resource organizations, a sharing model between local enterprises, and a sharing mode in different places within enterprise groups. Regarding the model of shared employees, there is currently no consensus. Based on the main elements of shared employees, this paper attempts to divide the model of shared employees in the hotel industry into four categories, namely, hospitality industry-to-hospitality industry $(\mathrm{H} 2 \mathrm{H})$, hospitality industry-to-employee (H2C), employee-tohospitality industry $(\mathrm{C} 2 \mathrm{H})$ and employee-to-employee $(\mathrm{C} 2 \mathrm{C})$, which can be further summarized into three categories, namely, shared employees at the hotel level, shared labor between individuals and hotels, and shared labor at the individual level. For the hotel industry, H2H/hospitality industry-to-platform-to-hospitality industry $(\mathrm{H} 2 \mathrm{P} 2 \mathrm{H})$ and $\mathrm{C} 2 \mathrm{H} /$ employee-to-platform-hospitality industry $(\mathrm{C} 2 \mathrm{P} 2 \mathrm{H})$ are the current mainstream shared employee models (cf. e.g. Figure 1).

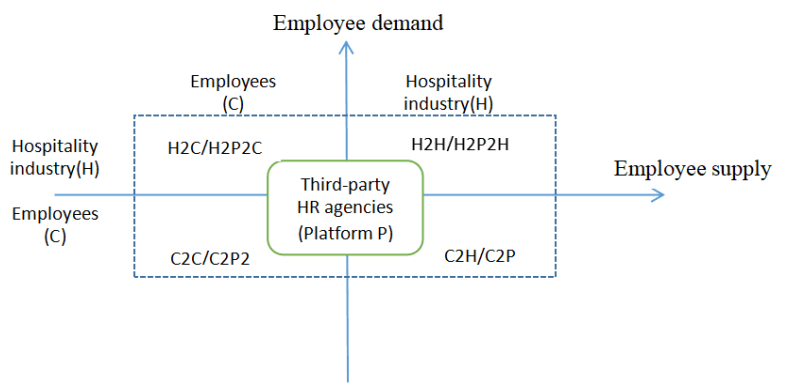

Figure 1: Shared employee base pattern framework

\subsubsection{Sharing of staff between hotels}

Employee sharing at the $\mathrm{H} 2 \mathrm{H}+\mathrm{H} 2 \mathrm{P} 2 \mathrm{H}$ enterprise level among the hotel industry, retail industry and hotel industry is the mainstream model under COVID-21. The model of cooperation includes direct cooperation, i.e., $\mathrm{H} 2 \mathrm{H}$, and indirect cooperation coordinated by a third party, i.e., H2P2H, which falls under the shared employee model at the enterprise level, as shown in Figure 1.

The $\mathrm{H} 2 \mathrm{H}$ model refers to the direct docking between a hotel and the pattern of sharing employees without any third party. The model is suitable for the special period. The hotel or hotel industry and other services between hospitality industry employees exhibit a large imbalance between emergency cooperation, generally have fixed joint partners, and have a certain cooperation basis and trust mechanism. The $\mathrm{H} 2 \mathrm{P} 2 \mathrm{H}$ model refers to relying on third-party human resource service organizations, hotel industry associations, artificial intelligence employment platforms and other third-party organizations to transfer job vacancy information, match and coordinate labor supply and demand, etc.

Based on the different sources of idle employees, hotel shared employees can be divided into three forms: cross-border shared labor among enterprises, shared labor within the hotel group and cross-functional shared labor among departments within a hotel. Among them, (1) the cross-border sharing of labor among enterprises is characterized by different natures of supply and demand in the industry and cooperation between both enterprises. For example, the cooperative labor model between the hotel industry and supermarkets under COVID-19 belongs to the cross-border sharing of labor in different industries and fields. Hotel wedding banquet organizations and wedding planning companies share employment. (2) In the group, shared labor refers to each actor within the same group with membership or a correlation between different subsidiaries, in which the group internally adjusts staff resources to ease a temporary shortage. For example, in the launch of the new Champions League Prosperous Group, the coordination between the Hefei and Anhui Fuyang Hotels, real estate, and the labor pension industry is a typical group with a pattern of shared employment. (3) The cross-functional sharing of labor among departments within a hotel refers to the cross-functional coordination of labor among different departments and positions within the same hotel. For example, "support for Chinese food" is provided by other departments of a hotel, such as the housekeeping department and concierge department, when the hotel undertakes large banquet reception tasks. Under COVID-19, only some multiskilled employees in resorts and hotels will be retained for multiple posts to reduce labor costs and to cope with the impact of COVID-19. As another example, in the new retail context, a hotel uses the e-commerce distribution model to realize the internal sharing of labor through a distribution platform. That is, under the condition of maintaining the original post functions unchanged, the nonsales employees within an enterprise can also cross-function to carry out distribution tasks and receive commissions.

\subsubsection{Individuals share employment with hotels}

$\mathrm{C} 2 \mathrm{H}+\mathrm{C} 2 \mathrm{P} 2 \mathrm{H}$ is a sharing model between employees and a hotel. Specifically, it refers to the sharing of individual labor by employees with a hotel $(\mathrm{C} 2 \mathrm{H}$ model). The operation mechanism is shown in Figure 1. It can be subdivided into two forms, $\mathrm{C} 2 \mathrm{H}$ and $\mathrm{C} 2 \mathrm{P} 2 \mathrm{H}$, based on whether there is a third-party intermediary platform involved. The $\mathrm{C} 2 \mathrm{H}$ and $\mathrm{C} 2 \mathrm{P} 2 \mathrm{H}$ staff sharing models refer to the sharing of individual 
employees through direct docking or connection methods such as a platform. Individual employees' own idle labor resources are shared by a hotel to provide the recruitment services to satisfy a business enterprise's need for a special type of work or temporary labor demand, for example, the paperless office and system management, the home office, and the remote office after the launch of the new Champions League. This approach is generally accepted, especially in special professions. It is common for accountants, legal staff and other positions to be shared among single hotels or small-scale catering industries, which is conducive to reducing human resource costs. Most star-rated hotels in China invite employees who are temporarily out of business to work for short periods through intermediary platforms to meet the demand for employment in busy and peak seasons. In this case, employees who are out of business are employed by hotels through third-party labor service companies, which falls under the $\mathrm{C} 2 \mathrm{P} 2 \mathrm{H}$ shared employee model.

There are four key points in the operation of shared employees in the hotel industry:

\section{(1) Labor relations}

Currently, $\mathrm{H} 2 \mathrm{H}$ sharing generally does not break the original employment relationship. Employees are supplied between hotels, and employees maintain individual long-term and stable labor relations. The employee needs the hotel, and individual employees mostly form a short-term labor relationship with hotels, constituting a labor relation of cooperation between the supply and demand of hotel staff. Personnel problems become the responsibility of the supply of hotel employees. The hotel provides temporary employment and basic income for employees on demand.

\section{(2) Salary payment}

The salary payment of hotel shared employees is mostly fixed in terms of hourly wages and piece rates. There are three payment methods: direct payment, payment to the original unit and payment on behalf of the original unit, and payment through a third-party human resource agency. In the practice of sharing employees in hotels, employees who are shared can generally obtain their wages from only one party, except outside the legal working hours of the original unit.

\section{(3) Agreement signing}

Internal sharing within a hotel is generally short-term sharing, mostly based on an oral agreement. For sharing within the group or between enterprises, a tripartite agreement can be signed after consensus to clarify the rights and responsibilities of the three parties, and some hotels entrust a third-party labor company to sign an agreement.

\section{(4) Work-related injury liability}

According to the relevant provisions of the Regulations on Work-Related Injury Insurance, if an industrial injury accident occurs during the period when an individual employee participates in employee sharing, the supplier of the employee is still responsible for the industrial injury insurance, while the supplier of the employee can recover compensation from the actual employment enterprise. In short, the individual rights and interests of the employee shall not be infringed upon.

\section{The Advantages of Sharing Employees in the Hotel Industry}

\subsection{Advantages for the Macro Environment}

We should respond flexibly to environmental changes and maintain social stability. For example, during the COVID-19 pandemic period, the model of sharing employees was implemented and exported to the retail industry, the logistics industry and other industries with a large demand for employees as the supplier of shared employees, which is conducive to the unified management of idle hotel employees, the reduction in human resource costs and the optimization of labor resource allocation. Based on the needs of society and the development of its own industries, sharing employees will promote the cross-industry transfer of idle labor, reduce the risk of industrial and large-scale unemployment, and promote the healthy and high-quality development of the social economy. The shared employee model is helpful for maintaining the ecological balance of the whole social and economic circle, promoting the reform of the labor market structure to be more flexible and shareable, and promoting the next round of iteration in employment relations.

\subsection{Advantages for the Hotel Enterprise Itself}

The shared employee model can be used as an effective supplement to the traditional employment model in the hotel industry. This model makes it possible to cope with the short-term sudden or seasonal demand fluctuations of the hotel, and it alleviates the extreme employment problems of "idle death" and "busy death". With the launch of the new Champions League and new retail, logistics and other services need to return to work and production, which involves difficult hiring. In contrast, a large number of employees in the tourism industry, such as the hotel industry, are out of business, which involves difficult jobs at home. The sharing model breaks the traditional employment model, which is theoretically based on the premise of a limit to the competitive strategy of the industry. Additionally, the model breaks through the original labor contract, which is based on fixed time work and fixed location elements. It effectively alleviates the imbalance between the supply and demand of labor resources and the polarization of labor demand in the special period.

At the same time, the model of shared hotel employees, in case of the actual needs of the business, will involve labor costs to pay, which will then save on the passive artificial cost and social cost of idle time. It can also reduce the hotel staff recruitment and training costs from the short-term surge in demand, improve the matching efficiency of human resources to the business requirements and response speed, realizing fast postemergency staffing. It will help promote the optimization of the hotel's labor structure, cross-border cooperation and innovation in human resource management.

It can attract and retain talent, avoid large-scale layoffs, reduce the employee turnover rate, stabilize labor relations, 
and provide a talent supply for the hotel's "rebirth from disaster" and long-term development.

Shared employment is the embodiment of the hotel industry's sense of social responsibility and the concept of serving people's livelihood, which is conducive to establishing a good social image, improving brand reputation, and boosting the high-quality and sustainable development of the hotel. At the same time, through cultural retention, emotional retention stimulates the loyalty of employees to the supply enterprise.

\subsection{Advantages for Individual Employees}

The shared employee model solves the problem of employment and increases income. For example, the launch of the new Champions League mostly involves the traditional restaurant and hotel tourism industries Most employees bear the economic costs of housing, children's schooling, and life stress. The shared staff model can provide a kind of new employment for closed employee selection and a "buffer", and it can help the short-term "unemployed" and ease the economic pressures of life.

Cross-boundary work under the shared employee model is conducive to employees' exposure to more types of work, learning more cross-boundary knowledge and working methods, and opportunities for cross-boundary learning and job rotation training. At the same time, it helps to stimulate the desire of workers to strengthen their skills learning and improve their own quality to enrich their own skills, enhance the accumulation of experience and promote an overall improvement in quality.

Under the shared employee model, employees are in the working state, which will help them enter the working state quickly after the end of shared labor and reduce the psychological impact of returning to work on employees. In addition, with the new generation of those born after the 1990s and after the 2000s and other employees becoming a new force in the workplace, they are more in pursuit of personal value and a rich career. "Amphibian youth" and "slash youth" have also become symbols of the new generation of employees. Thus, the shared employee model may be a possibility in pursuing freedom and the ideal career.

\section{The Challenges of the Hotel Industry Sharing its Employees}

\subsection{The Challenges of Hotels Sharing their Staff}

\subsubsection{Challenges at the national and social levels}

Relevant laws and regulations need to be improved. At present, the national legal regulation obviously lags behind the practice of enterprise employment sharing. As a result, there is no clear legal basis for enterprise labor relations or for the division of the rights and responsibilities of both parties, the protection of workers' rights and interests, professional title assessment, etc. The relevant laws and regulations of shared employment urgently need to be improved. At the same time, the traditional labor contract and labor employment system are mostly based on the standard employment model, while wage settlement, post standard determination, labor employment risk sharing, illegal labor dispatch and profit-making behavior under the shared employment model are in urgent need of relevant policy guidance.

The guiding role of local governments and industry associations and the lack of a shared employee platform lead to difficulties in matching the supply and demand of the surplus and shortage of staff. The current shared employment model mainly relies on some sporadic but not large-scale system docking models between enterprises to operate. Labor surplus and shortage are difficult to match accurately and in a timely manner. Large-scale replication and popularization are difficult.

\subsubsection{Challenges at the hospitality industry level}

With the increasing popularity of new forms of diversified employment such as shared staff, hotel human resource management will also face more complex and diversified challenges, including shared labor's highly flexible liquidity, the pursuit of stability and a sense of belonging. The model conflicts with the idea of traditional management, and employees across different organizations and management systems (such as recruitment, evaluation and rewards and punishments) will face challenges. The shared weight division and management system for both labor enterprises need to be remodeled. There is a cross-border talent competition, and some employees may prefer the new enterprise culture, leading to brain drain, which will complicate labor relations. As a result, the responsibility and rights of all parties sharing labor are not clearly defined, and the category of shared employees (such as the type of work, post, responsibility, cycle) must be defined.

There are challenges of employment risk that need to be defined. They include employee industrial injuries, the dilution of corporate culture and employee loyalty, post adaptability, team working, employee health and safety, employee welfare, skills match, service standard guarantee, business competition, business risk of leaks, the guarantee of rights and interests, contract signing, labor disputes and employee discipline, illegal labor dispatching risks, payment by employees, salary payment, social security problems, individual income tax, the division of rights, responsibilities and benefits among enterprises, and the unclear definition of employment relations.

There are labor quality and cost challenges. For example, when the hotel is busy, the entry threshold of shared employees is lowered, people and post skills are not matched, and the quality of employees is uneven. As a result of these problems, it is difficult to match people and posts and to guarantee the stability of product and service quality. Most shared employees can engage in only simple and low-skill manual labor, and the service standard realized by shared employees can only be without dissatisfaction, and the satisfaction state is limited.

After the sharing of labor, the supplier of employees will be faced with the risk of difficulty in guaranteeing the desire of employees to return and brain drain, and it may be difficult to respond quickly to a change in their own labor demand. The 
borrower of employees will also face challenges such as a difficult recovery of talent training costs and loss of human capital (such as employees' knowledge, skills and work experience) (De Stefano F, Bonet R, Camuffo A. 2019: 979-1002), business fluctuations caused by the return of shared employees, and labor surplus caused by the return of old employees.

\subsubsection{Challenges at the individual employee level}

The shared employee model poses new challenges to the comprehensive abilities of individual employees in various ways, such as their work skills, role transformation and environmental adaptation, and it requires individual employees in the tourism industry to have a "specialized and versatile" skill structure. At the same time, individual cross-functional employment and internal shared employment may also affect the quality of individual employees' own work.

In the shared employee model, the individual employee is more in a passive and weak position and plays the role of an informal employee, a noncore position, and an auxiliary and low-skilled job in the employee input enterprise. In turn, individual employees are faced with many challenges, such as promotion channels, career development, annual performance appraisal, a lack of a sense of belonging and psychological security, and retention problems after the end of the individual's shared identity.

There are challenges in terms of the legitimate rights and interests of employees, wages, benefits and security. Additionally, there are other challenges, such as individual employees illegally earning dispatch fees from companies, different pay for the same work, and the risk of work-related injury caused by unskilled cross-border work. At the same time, the shared employee model will directly change the working position, working place, working content and salary of workers, which will lead to challenges in terms of deliberately lowering the salary and working with higher intensity.

\subsection{The Measures of Hotel Shared Employees}

\subsubsection{Countermeasures at the national and social levels}

We will improve laws and regulations, strengthen the appropriate supervision and policy-leading role of the "visible hand" of government departments, and explore mechanisms for standardizing and normalizing the sharing of employees. The current shared employee model is mostly based on the spontaneous behavior of enterprises. Government departments and industry associations can improve and implement employee sharing rules as soon as possible. We need a shared staff pattern matching scope, old and new unit labor property treatment, the definition of the norms and the implementation of the contract of employment, labor relations dispute handling, employee career development and rights protection and so on to provide more perfect policies and regulations to guide and promote the sharing of flexible employment groups to implement the legal rights and interests of employees at the legal level. We should ensure that there are laws for sharing employment, combat abuse and illegal profit making, address the concerns of all parties and protect basic rights and interests.

We should set up and improve staff sharing platforms and establish a "reservoir" of human resources. Government departments can be set up a staff sharing platform across provincial, industry, and many other levels, encourage and guide enterprises to use docking platform release information to adjust labor, and realize the dynamic monitoring of the project we adopted and accurate matching. The Hefei municipal government and relevant departments should jointly promote the establishment of a staff sharing platform, enterprise alliances and the project we discuss. At the same time, trade unions, trade associations, human resource service organizations and other social forces should give full play to the intermediary and guarantee role in employment adjustment, and vigorously support and promote the creation of more organizations.

We will intensify efforts to popularize new forms of employment and concepts and strengthen individual skills education and training as well as the protection of workers' rights and interests. The government can invest more money to guide workers in lifelong learning, cross-industry and multiskill learning and training and constantly improve their employability to ensure that employees can accept and adapt to the new form of employment more quickly. In addition, the government should re-examine the traditional social security system of workers based purely on labor relations, strengthen the protection of the individual rights and interests of shared employees, and explore the construction of a new social security system that is more diversified and based on work transactions instead of purely based on work relations to "provide basic income for all".

\subsubsection{Coping strategies at the hotel level}

Hotels should change the traditional human resource management concept, change the hotel industry organization and employment structure, explore the standardization and normalization of shared employees and improve the employment mechanism and system of supporting rules. First, an enterprise alliance of shared employees should be established to boost the normal development of shared employees through the enterprise alliance. Second, partners should avoid the phenomenon of homogenization, realize the sharing and deployment of talent resources between different enterprises in the peak and low seasons, and pay attention to the sharing of employees among positions with similar types of work to reduce the training costs brought by cross-enterprise employment and to ensure the quality of employment. Additionally, hotels should fully understand their employment needs, accurately match the employment resources, improve the selection standards for shared employment, develop a flexible exit mechanism and smooth the rapid return channel of employees, and enhance the sense of belonging, loyalty and trust of employees by improving the cooperative employment agreement, strengthening the welfare care of employees and post transfer assistance, etc. They should strengthen the construction of corporate culture and create a mobile, open, long-term first corporate culture to ensure that employees can return to work in a timely manner and reduce brain drain. Furthermore, hotels should assess the 
feasibility and expected risks of sharing employees in combination with the hotel's own talent pool and strategic development and fully measure the proximity of the business model of both parties, the quality and skill span of employees, the business competition relationship, and their own management ability.

Both parties shall clarify the cooperative employment agreement and reasonably avoid risks to achieve an effective balance between the employment cost, risks and economic benefits of the enterprise. Exporting enterprises, importing enterprises and employees shall sign a written agreement through negotiation, including the cooperation agreement between the two parties of employee-enterprise cooperation and the labor relationship arrangement agreement between the enterprise and employees, to clarify the ownership of rights, responsibilities and benefits of labor cooperation between the parties. Additionally, they should follow the existing laws and explore the establishment of appropriate employment relations (such as part-time or secondment), and with the help of a third-party platform or by signing relevant agreements, they should protect the rights and interests of employees, thereby reducing employment risks and improving matching efficiency.

Hotels should set up an internal sharing employment platform, make full use of artificial intelligence technology to empower, and actively seek help from the government, third-party human resource service agencies, industry associations and other third-party shared employee platforms. First, hotels can build an internal shared employment platform, which can not only reduce the unstable factors of flexible employment but also fully mobilize the enthusiasm of employees and tap and stimulate the potential of employees. Second, hotels can seek help from the government, third-party human resource service agencies, industry associations, and so on to more efficiently match the demand of labor surplus and shortage. Third, a shared employment platform enabled by artificial intelligence technologies such as Big Data, Artificial Intelligence and Blockchain can realize the accurate prediction of labor hours and the number of employees required by enterprises, carry out the online matching of flexible demand and use online video to complete the interview process. In addition, artificial intelligence technology and platforms can be used to manage and share employees to enhance the flexibility of the employment structure, organizational flexibility and anti-vulnerability ability.

\subsubsection{Coping strategies at the individual level of employees}

It is important to practice lifelong learning and enhance the comprehensive ability of slash employment. Individual employees should not only improve their professional skills but also pay attention to improving complex cross-border skills, pursuing a wider field of work, being a number of omnipotent "slash talent" and always being prepared to deal with the external risk and uncertainty of the "ABZ plan" (that is, the main business, sideline production and "save project"), thus enhancing their employment competitiveness and risk resistance, improving their individual income and ensuring sustainable employment opportunities (cf. e.g., Wilmers, N. 2020).
Individual employees should enhance their self-protection consciousness, actively understand and share employee rules through legal means, trade unions, industry associations and other channels, and safeguard their legitimate rights and interests in accordance with the law (Zhang Chun'e, 2021: 013-015). In addition, individual employees should take advantage of various types of artificial intelligence shared employment platforms to fully display their talents and explore employment opportunities.

\section{Conclusions and Prospects}

Beginning with an analysis of the shared employee model in the hotel industry, this study discusses the advantages, challenges and coping strategies of employee sharing in the hotel industry in the postpandemic period. It finds that employee sharing is a strategically innovative model of human resource management in the hotel industry for realizing flexible employment. The implementation of the shared employee strategy constitutes a new form of win-win cooperation between hotels, society and staff, and it is an effective practice for hotels to reduce costs and increase efficiency, raise employment and employee incomes, and stabilize the social economy. Hotel shared employment also faces a series of bottlenecks and challenges, such as the imperfect legal system.

With the deepening of practice and theoretical research related to the shared employee model in the hotel industry, this study suggests two research prospects: cross-enterprise management and employee relations in the process of shared employment on the demand side and the question of how the supplier of shared employees guarantees the shared employee return rate. At the same time, the adaptability of the proportion of shared employees in the hotel industry also requires in-depth future research.

\section{Acknowledgement}

This study is supported by the projects: Key Project of Humanities and Social Science of Education Department of Anhui Province (SK2020A0312); New Liberal Arts, New Medical Research and Reform Practice Project (2020WYXM128); Excellent talents education and training plan 2.0 project (2020zyrc03); Quality Engineering Teaching Research Project of university (2019JYXM15).

\section{References}

[1] Chen Xiangdong, Zhang Feng, Paul M.A. Baker. Gig economy and sharing economy: reengineering and promotion of labor[J]. China Soft Science, 2020 (1): 21-29.

[2] De Stefano F, Bonet R, Camuffo A. Does losing temporary workers matter? The effects of planned turn-over on replacements and unit performance[J]. Academy of Management Journal, 2019, (4): 979-1002.

[3] Feng Xiliang, Zhang Jianguo, Zhan Jing, et al. Flexible employment: talent is my own to talent for my use[M]. Beijing: China Renmin University Press, 2018. 
[4] Friedman G. Workers without employers: Shadow corporation sand the rise of gig economy[J]. Review of Keynesian Economics, 2014, 2(2): 171-188.

[5] Guo Z G. The boundary-less career balance based on employability[J]. Economic Management, 2007(19): 60-64.

[6] He Jiang, Yan Shumin, Guan Jiao. What exactly is a shared employee? - Origin, connotation, framework and trend[J]. Business Research, 2020, 6: 1-13.

[7] Petriglieri G, Ashford S J, Wrzesniewski A. Agony and ecstasy in the gig economy: Cultivating holding environments for precarious and personalized work identities[J]. Administrative Science Quarterly, 2018, (1): 124-170.

[8] Wilmers, N. Job Turf or Variety: Task structure as a source of organizational inequality $[\mathrm{J}]$. Administrative Science Quarterly, 2020.

[9] Zhang Chun'e. Challenges and countermeasures of human resource management in the hotel industry in the Post-epidemic Era[J]. China-Arab States Science and Technology Forum, 2021(6): 013-015.

[10] Zhou Xuan. "Shared Staff", A new way of hotel self-help?[J]. China Conference and Exhibition (China Conference), 2021, 12: 26. 